\title{
Influencia del diseño del espacio en los procesos de enseñanza-aprendizaje. Revisión
}

\section{Space design influence in the teaching-learning Processes. A review}

\author{
Jesús Miguel Muñoz-Cantero*, Ricardo García-Mira** y Vicente López-Chao*** \\ Universidade da Coruña \\ *Departamento de Filosofía y Métodos de Investigación en Educación, Grupo GIACE, **Departamento de Psicología, Grupo de \\ Investigación Persona-Ambiente, GLAMURS, ${ }^{* * *}$ Grupo GIACE
}

\begin{abstract}
Resumen
La investigación sobre factores relativos al diseño de espacios educativos ha crecido en los últimos años, sin embargo se siguen manteniendo principios tradicionalistas en la gestión de las aulas. La inversión en un elemento común a los estudiantes, como son los factores del diseño del espacio educativo, puede significar un desarrollo cultural a gran escala. Como consecuencia de esto, dado el coste económico, la permanencia en el tiempo de las edificaciones educativas y la situación de necesidad de proyectos de rehabilitación y o conservación de muchas de éstas, se hace necesario comprobar el posible impacto del diseño del espacio en cuestión de mejora educativa. Tras una primera aproximación se concluye que entre los factores del diseño del espacio que inciden en el proceso de enseñanza-aprendizaje se encuentran la iluminación, la acústica o el nivel térmico entre otros.
\end{abstract}

Palabras clave: Espacio educativo, Diseño del espacio, Diseño de aula

\begin{abstract}
Research on factors related to the design of educational spaces has grown in recent years, yet still maintaining traditionalist principles in classroom management. Investing in an element that is common to all students, such as design factors of educational space can mean large-scale cultural development. As a result, given the economical cost, the retention time of educational buildings, and the situation of need for rehabilitation or conservation of many of those, it is necessary to test the possible impact of the space design as a matter of improving education. After a first approximation, it is concluded that the factors of space design that affect the process of teaching and learning are lighting, acoustics, and thermal level, among others.
\end{abstract}

Keywords: educational space, space design, classroom design.

\section{Estado del arte}

La investigación sobre factores relativos al diseño de espacios educativos ha crecido en los últimos años. En 1955 Joseph Gottler fue uno de los primeros en hablar acerca de los factores que influyen en el aprendizaje formulando su ley pedagógica fundamental, en la cual menciona que el trabajo educativo mejorará sus resultados cuanto mayor sea la armonía entre educadores y los factores influyentes. Otros autores han demostrado que los factores en el entorno construido afectan a la retención, a la atención, a la motivación, al aprendizaje y al rendimiento académico (Durán-Narucki 2008; Scott-Webber, Strickland y Kapitula, 2013).

Sin embargo a pesar de los estudios existentes, se siguen manteniendo principios tradicionalistas en la gestión de las aulas. Esta creciente generación de información y la necesidad de nuevos modelos o patrones de diseño, da lugar a este trabajo para facilitar una información clara y concisa sobre qué factores del diseño del espacio se deben tener en cuenta a la hora de proyectar un espacio educativo.

La importancia de identificar los factores del entorno de aprendizaje reside en su influencia en los comportamientos, actitudes y mejora de logros por parte del alumnado. El propósito de este trabajo es indagar sobre los factores del diseño del espacio educativo que influyen en el alumnado a través de una revisión de la bibliografía existente sobre esta temática, haciendo así más fácil su búsqueda para los profesionales que requieran conocimientos sobre la temática. Fundamentalmente, la problemática a la que se enfrentan los investigadores es la inalterabilidad de los espacios educativos y la discordancia con las nuevas metodologías exigidas por el Espacio Europeo de Educación Superior (EEES).

\section{Condicionantes del entorno educativo en el proceso de aprendizaje}

\section{Factores ambientales}

Nuestros sistemas emocionales han ido evolucionando en respuesta al medio natural, por lo que parece lógico afirmar que los factores ambientales o naturales deben influir en el proceso de diseño de las aulas (Barret, Zhang, Moffat y Kobbacy, 2013), los cuales influyen directamente en el confort de los estudiantes o en el bienestar y consecuentemente en comportamientos o en resultados de aprendizaje. Se incluyen por lo tanto:

- La luz: que facilita el rendimiento visual.

- Sonido: condiciones necesarias para escuchar el sonido deseado y no los desagradables. 
- Temperatura: mantenimiento del equilibrio en la temperatura corporal

- Calidad del aire/ventilación: humedad, elementos contaminantes, olores, etc.

\section{Iluminación}

Uno de los objetivos generales de la iluminación en un espacio de aprendizaje es proporcionar un entorno visual que apoya el proceso de aprendizaje, tanto para los estudiantes como para los profesores (Rea, 2000) Si la iluminación es demasiado brillante o si presenta un color desagradable puede distraer al alumno que trata de leer (Burrus, 2001). Es necesario, no solamente por cuestiones de economía energética, el equilibrio entre iluminación natural y artificial. La luz natural no solamente ayuda a la capacidad visual sino que posee una calidad suave y difusa de la que la iluminación artificial carece. Por ello, los techos bajos y las aulas profundas pueden causar que los alumnos experimenten sentimientos sombríos por la disparidad de la luz. Se ha observado que personas que carecen de luz natural alcanzan menos bienestar emocional y un descenso de las hormonas que regulan el ciclo del sueño. Por otra parte, una exposición prolongada a la iluminación artificialeleva la excitación del sistema nervioso causando estrés en el alumnado (Küller y Wetterberg, 1993). Hesching Mahone Group (1999) encontró correlaciones positivas entre las variables, verificando que los estudiantes progresaron un $20 \%$ más rápido en matemáticas y un $26 \%$ más en lectura que los que tenían menos luz; también la creación en los alumnos de una sensación física y mental de confort. De esta manera, para obtener una buena iluminación habría que tener en cuenta: la cantidad de luz, la distribución correcta de sus componentes y buscar la ausencia de reflejos. Otros beneficios de la luz del día es ayudar al alumnado a retener y aprender la información dada (Rittner y Robbin, 2002). Winterbottom y Wilkins (2009) dan un mayor énfasis a la importancia de la luz diurna evitando y dejando la luz artificial para las horas del día que lo requieran.

\section{Acústica}

En el ámbito del sonido el objetivo principal es mantener las condiciones adecuadas para la reproducción y recepción de los sonidos buscados y deseados, por lo que la calidad de la percepción auditiva es un aspecto fundamental. La ausencia de ruido unido a la buena calidad de la percepción desemboca en una mejora de la comunicación y del aprendizaje. Por ello los requisitos necesarios para una buena acústica en el ámbito del aprendizaje serían: nivel de ruido aceptable, niveles de sonido óptimos y correcta distribución de los alumnos en el aula. Una acústica pobre o mala en las clases puede crear un entorno de aprendizaje negativo para muchos estudiantes. El diseño acústico resulta imprescindible para prevenir distracciones por los ruidos causados en los ambientes de aprendizaje, es decir, que minimicen el ruido de los sistemas de aire acondicionado, equipos o aulas contiguas (Torbert,
1987). Existen dos causas de ruido en el aula: el externo (coches, aviones, etc.) y el interno (ruido generado por alumnos y profesores) (Rivlin y Weinstein, 1978). El ruido es un factor importante para evaluar las aulas porque está relacionado con el logro, la cognición espacial y la densidad; además Klatte, Hellbruck, Seidel y Leistner (2010) realizaron una investigación donde encontraron que las condiciones acústicas en las aulas no se ajustaban en la mayor parte de los casos a las necesidades de los oyentes. Examinaron también el efecto de la reverberación del sonido sobre el rendimiento y bienestar de los alumnos, demostrando que la acústica, la mayor o menor reverberación, puede tener un efecto negativo o positivo en la percepción del alumnado, de sus profesores y compañeros, así como en su rendimiento académico.

\section{Nivel térmico}

Otro de los factores naturales a los que se aludían anteriormente era la temperatura, donde el ambiente térmico afecta a la sensación de "caliente" o "frío" y "húmedo" o "seco" de los ocupantes del aula (Huang, Yingxin, Ouyang y Cao, 2012).El confort térmico se logra a través del mantenimiento de la temperatura, la humedad y el movimiento del aire y las condiciones de la actividad humana en un determinado rango. Para la obtención de ese punto medio térmico hay tres condicionantes: la orientación, la disposición de la construcción y la colocación de las ventanas. De tal manera, que las instalaciones deben ser diseñadas con un ais lamiento suficiente para evitar cambios bruscos de temperatura en el interior debido a las condiciones extremas (Flynn, Kremers, Segil y Steffy, 1992). En este aspecto, hay tres cuestiones sobre la comodidad del alumno en relación al ambiente térmico de las aulas: en primer lugar, que las condiciones térmicas de los alumnos son diferentes a la de los profesores, en segundo, la tensión inducida por vía térmica, que puede alterar el aprendizaje $\mathrm{y}$ en tercer lugar, que la temperatura afecta a la capacidad de resolución de problemas; por lo tanto, las aulas deben estar diseñadas de manera que los alumnos y profesores puedan controlar la temperatura, haya un buen movimiento de aire y cierta cantidad de humedad (Harmon, 1953). Por otra parte, en cuanto al uso del termostato central, se realizó un estudio en 23 escuelas gallegas, determinando un efecto negativo sobre el uso del mismo, pues ni todas aulas eran iguales ni tenían la misma orientación y número de alumnos (Crespo y Pino, 2007). No obstante, con anterioridad se había sugerido que en las aulas de educación primaria los profesores llevasen chaqueta o jersey pues los niños son más activos y tienen un metabolismo acelerado por lo que se encuentran más cómodos con menos ropa (McVey, 1969); pues las temperaturas más frías se asocian con un mayor nivel de confort, productividad y concentración del alumnado más joven (King y Marans, 1979). En cualquier caso, si el espacio de aprendizaje presenta de manera continua un nivel térmico anormal o incómodo, los estudiantes pueden llegar a optar por no utilizar dicho espacio (Burrus, 2001). 


\section{Ventilación}

Otro de los factores fundamentales en la actualidad es la calidad del aire,pues las malas condiciones de ventilación internas, el aire fresco limitado y el uso de alfombras, genera un ambiente ideal para los ácaros y para dañar el aire de la habitación. Siendo elementos problemáticos en la comodidad y en la salud. Coley y Greeves (2004) realizaron un estudio en este aspecto investigando las bajas tasas de ventilación y su efecto en las funciones cognitivas de una clase de primaria. Se utilizaron una batería de pruebas estandarizadas demostrando que los procesos de atención de los niños en edad escolar era significativamente más lento cuando el nivel de $\mathrm{CO} 2$ (medidor de los niveles contaminantes usados en el estudio) era elevado. Hablaron de una disminución de un 5\%. La calidad del aire interior y la ventilación adecuada están estrechamente vinculados y se encuentra como un problema común en las escuelas. Bakó-Biró, Kochlar, Clements-Croomel, Awbi y Williams (2007) recalcaron que en los últimos estudios que se hicieron sobre la calidad del aire en la mayor parte de los países el promedio de $\mathrm{CO} 2$ en las aulas superaba la tasa límite, de tal manera que no sólo la salud o el confort del alumno se ve perjudicado, sino que su rendimiento académico se ve alterado junto a los otros dos elementos. También realizó una investigación en una serie de escuelas al sur de Inglaterra en el que pudo determinar que los niveles de $\mathrm{CO} 2$ superiores al $1 \%$ afectaban a la salud de los alumnos, como ya habían expresado investigaciones médicas, con dolores de cabeza, mareos, etc... Lo que afectaba de manera negativa al rendimiento académico de los alumnos. Por el contrario, determinó que en las aulas correctamente ventiladas había habido un impacto significativo en la mejora del rendimiento escolar, siendo aún mejor la influencia en los alumnos con habilidades matemáticas superiores, pues su rendimiento se vio incrementado un $7 \%$ bajo las condiciones de ventilación mejoradas.

\section{Factores espaciales}

\section{Organización espacial}

El espacio está implicado de manera directa en la creación y mantenimiento de "la escuela", pudiendo ser vis ta como una constelación de las relaciones en curso y no como un lugar predeterminado (Nespor, 1997). La ordenación de las aulas, laboratorios, salas de profesores $\mathrm{y}$ patios de recreo en las escuelas secundarias determinan el mantenimiento de ciertas relaciones de poder de tal manera que la exploración de la espacialidad de las escuelas es una forma de poner en relieve dichas relaciones.Un buen diseño del aula permite más interacciones positivas entre profesores y alumnos, de esta manera se reduce la probabilidad de que ocurran comportamientos problemáticos (Martella, Nelson y Marchand-Martella, 2003). Aunque las modificaciones del diseño se utilizan de manera preventiva, Guardino y Fullerton (2010) muestran que a través de las modificaciones del entorno del aula se puede incrementar el compromiso académico y reducir los comportamientos disruptivos. Resulta determinante entonces que el diseño de las aulas proporcione flexibilidad para anticiparse a los cambios de los objetivos pedagógicos y a los programas educativos que se reflejan en las estrategias de organización (Barret y Zhang, 2009). En el ámbito de la privacidad ocurre que las personas pueden controlar mejor sus interacciones con otros, mientras que los espacios abiertos pueden provocar que este control sea algo complejo; por tanto, los espacios abiertos y flexibles alientan el concepto de comunidad, pero imposibilitan el de la privacidad (Leiringer y Cardellino, 2011).

El estudio de percepción del alumnado sobre el ambiente de la clase de La Roque (2008) mostró que conforme aumentaba el nivel académico, el nivel de disconformidad con el entorno también crecía. Un resultado preocupante según el mismo estudio es que a mayor dificultad de comprensión del entorno, los logros académicos eran más bajos.

El modelo de organización escolar más presente en la actualidad todavía sigue cánones centrados en el profesorado, y en el trabajo individual por parte del alumnado. Las relaciones de poder están inscritas en ciertos espacios dentro del aula, como en la situación de la mesa del profesor, símbolo de poder y punto de vigilancia (McGregor, 2004). Sin embargo para maximizar el uso o el diseño de la organización espacial es necesario atender a la metodología de enseñanza (Taylor, 2009). En cualquier caso, lo fundamental es que la organización espacial permita incrementar diferentes experiencias didácticas en el aprendizaje; por ello, Bautista y Borges (2013) proponen la flexibilidad de la disposición física como uno de los principios que deberían regir los espacios de aprendizaje, esto unido a otros principios como la adaptabilidad, lo que conlleva que el espacio se pueda adaptar a las necesidades de los alumnos y la multiplicidad, la cual se refiere a que este tipo de aulas presentarán características que permitan el uso de diversos tipos de recursos y estímulos.

\section{Mobiliario}

El ser humano pasa un tercio de la vida sentado, y en el caso del aula no es distinto. Sin embargo, Knight (1999) notificó que a pesar de la cantidad de horas que los estudiantes pasan en el aula el mobiliario escolar no había recibido la atención necesaria. Durante ese periodo académico tan largo, el estudiante adquiere una serie de hábitos posturales complicadas de cambiar en el futuro. Por lo tanto, resulta lógico tratar de diseñar el mobiliario para su máxima durabilidad, por lo que el mismo debería tener un diseño modular con partes intercambiables de manera que el diseño funcional que se desea se pueda ensamblar a través de un banco de partes (Al-Harkan, Ramadan, Sharaf y Helmy, 2013).

Además, generalmente el estar sentado durante un largo periodo de tiempo puede desembocar en diferentes problemas para la salud como dolores de cabeza, de cuello y lumbares (Molenbroek, Kroon-Ramaekers y 
Snidjers, 2003), desórdenes musculo-esqueléticos y déficit de atención (Bendix, 1987). Estos problemas en la salud del alumnado, pueden estar relacionados con el desajuste entre las medidas del estudiante y el mobiliario escolar (Parcells, Stommel y Hubbard, 1999). Por tanto para elaborar un diseño más apropiado de cualquier tipo de equipamiento es necesario la información antropométrica (Mirmohammadi, Houshang, Somayyeh, y Mostaghaci, 2011).

La principal función del mobiliario es dar soporte al alumnado durante las actividades cotidianas desarrolladas en el aula, requiriendo las mismas distintas posturas. Sin embargo, otra función de este mobiliario era minimizar el movimiento de los alumnos para facilitar vigilancia del profesor (Al-Harkan, Ramadan, Sharaf y Helmy, 2013); lo anterior se presenta como una muestra más del modelo de enseñanza centrado en el profesor. Muchos de los diseños de aprendizaje activo suponen que la mesa o el podio del profesor será móvil y estará colocada hacia el centro de la habitación, mientras que las mesas de los estudiantes estarán pensadas para fomentar la interacción en grupo siendo redondas, rectangulares o de forma octogonal incorporando pantallas u otro tipo de dispositivos tecnológicos; sus sillas serán también con ruedas para facilitar el movimiento (Painter, Fournier, Grape, Grummon, Morelli, Whitmer y Cevetello, 2012).

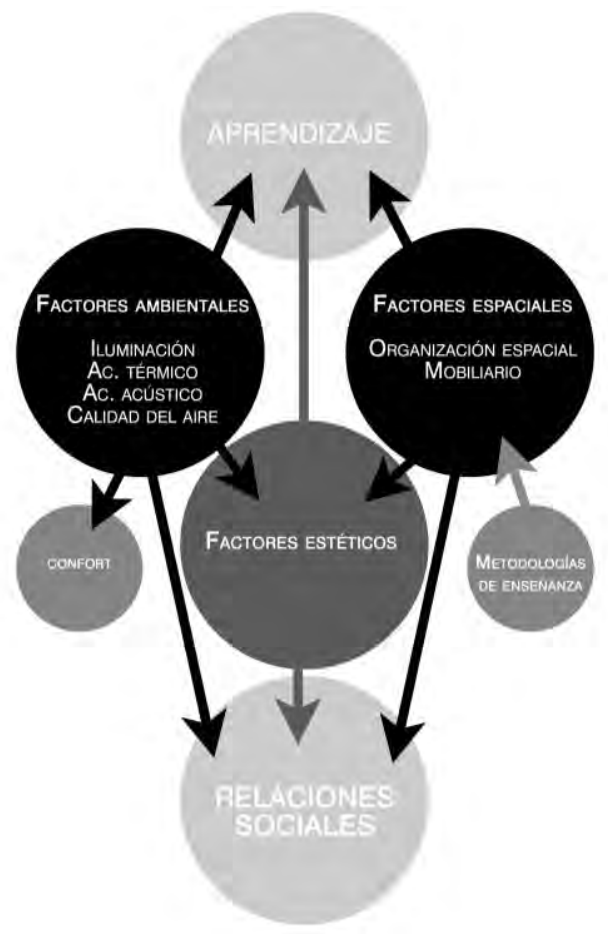

Figura 1. Elaboración propia

\section{Conclusiones}

Una vez analizados todos estos estudios, podemos concluir que existen determinados factores que ciertamente influyen en la mejora del proceso de aprendizaje como son: los factores ambientales y los factores espaciales.

Es importante el estudio de estos factores porque contribuye a ampliar la comprensión de su impacto en el proceso de aprendizaje. Se ve necesario establecer un modelo integral que tenga en cuenta las diferentes variables.

Aunque hemos trabajado estos elementos sería necesario trabajar otros como los diferentes componentes de un factor estético que aporte al espacio capacidad de atracción al público objeto.

\section{Referencias}

Al-Harkan, I., Ramadan, M., Sharaf, M. y Helmy, H. (2013). Designing a new school furniture suitable for Saudi Students. Engineering and Technology, 74, 4148.

Bakó-Biró, Z., Kochhar, N., Clements-Croome1, D.J., Awbi, H.B. y Williams, M. (2007). Ventilation Rates in Schools and Learning Performance. Proceedings of Clima 2007 WellBeing Indoors.

Barret, P. y Zhang, Y. (2009). Optimal learning Spaces. Design implications for Primary Schools. SCRI Research Report, 2, 2-55.

Barret, P., Zhang, Y., Moffat, J y Kobbacy, K. (2013). A holistic, multi-level analysis identifying the impact of classroom. Building and Environment, 59, 678-689, http://dx.doi.org/10.1016/j.buildenv.2012.09.016

Bautista, G. y Borges, F. (2013). Smart Classrooms: Innovation in formal learning spaces to transform learning experiences. Bulletin of the IEEE Technical Committee on Learning Technology, 15(3), 18-21.

Bendix, T. (1987). Adjustment of the seated work place with special reference to heights and inclinations of seat and table. Dan Med Bull, 34(3), 125-139.

Burrus, W. J. (2001). Adult learning environments: The relationship of Light and Colour in the Ambient Environment. The Journal of Continuing Higher Education, 49(3), 28-33, http://dx.doi.org/10.1080/07377366.2001.10400439

Coley, D.A. y Greeves, R. (2004). The effect of low ventilation rates on the cognitive function of a primary school class. Report R102 for DfES, Exeter University.

Crespo, J. y Pino, M. (2007). Description of environmental factors in schools. Lessons from study in north-west Spain. Review of Education, 53, 205218.

Durán-Narucki, V. (2008). School building condition, school attendance, and academic achievement in New York city public schools: a mediation model. Journal of Environmental Psychology, 28(3), 278-286. 
Flynn, J. E., Kremers, J. A., Segil, A. W. y Steffy, G. R. (1992). Architectural interior systems: Lighting, acoustics, air conditioning (3rd ed.). New York: Van Nostrand Reinhold.

Gottler, J. (1955) Pedagogía sistemática. Barcelona: Herder.

Guardino, C y Fullerton, E. (2010). Changing behaviours by changing the Classroom Environment. Teaching Exceptional Children, 42(6), 8-13.

Hall, I. y Higgins, S. (2005). Primary school students' perceptions of interactive whiteboards. Journal of Computer Assisted Learning, 21(2), 102-117, http://dx.doi.org/10.1111/j.1365-2729.2005.00118.x

Harmon, D. (1953). Controlling the thermal environment of the coordinated classroom. Minneapolis: Honeywell Regulator Company.

Heschong Mahone Group (1999). Daylighting in Schools. An investigation into the relationship between daylight and human performance. Detailed Report. Fair Oaks, CA.

Huang, L., Yingxin, Z., Ouyang, Q. y Cao, B. (2012). A study on the effects of thermal, luminous and acoustic environments on indoor environmental comfort in offices. Building and environment, 49, 304-309, http://dx.doi.org/10.1016/j.buildenv.2011.07.022

Klatte, M., Hellbruck, J., Seidel, J. y Leistner, P. (2010). Effects of classroom acoustics onperformance and well-being in elementary school children: A field study. Environmentand Behavior, 42(5), 659-692, http://dx.doi.org/10.1177/0013916509336813

King, J. y Marans, R. (1979). The physical environment and the learning process: A survey of recent research. Institute for Social Research.

Knight, N. (1999). Children's behaviour and the design of school furniture. Ergonomics 42(5), 747-760, http://dx.doi.org/10.1080/001401399185423

Küller, R. y Wetterberg, L. (1993). Melatonin, cortisol, EEG, ECG and subjective comfort in healthy humans: impact of two fluorescent lamp types at two light intensities. Lighting Research and Technology. 25, 7181.

La Roque, M. (2008). Assessing the perception of the environment in elementary Classrooms: the link with achievement. Educational Psychology in Practice, 24(4), 289-305,

http://dx.doi.org/10.1080/02667360802488732

Leiringer, R y Cardellino, P. (2011). Schools for the twenty-first century: school design and educational trans formation. British Educational Research Journal, 37(6), 915-934,

http://dx.doi.org/10.1080/01411926.2010.508512

Martella, R.C., Nelson, J.R. y Marchand-Martella, N.E. (2003). Managing disruptive behaviours in the schools. Boston, MA: Allyn \& Bacon.

McGregor, J. (2004). Space, Power and the Classroom. Forum, $46(1)$,

13-18, http://dx.doi.org/10.2304/forum.2004.46.1.2

McVey, G. (1969). Environment of learning: The application of selected research to classroom design and utilization. National Education Association.
Mirmohammadi, S.J., Houshang, M Somayyeh, J. y Mostaghaci, M. (2011). An assessment of the anthropometric data of Iranian University Students. International Journal of Occupational Hygiene, 3(2), 85-89.

Molenbroek, J. F., Kroon-Ramaekers, N. y Snidjers, C.J. (2003). Revision of the design of a standard for the dimensions of school furniture. Ergonomics 46(7), 681-694, http://dx.doi.org/10.1080/0014013031000085635

Nespor, J. (1997). Tangled up in School: politics, space, bodies, and signs in the educational process. New Jersey: Lawrence Erlbaum.

Painter, S., Fournier, J., Grape, C., Grummon, P., Morelli, J., Whitmer, S. y Cevetello, J. (2012). Research on learning space design: Present state, Future Directions. Report from the Recipients of the 2012 Perry Chapman Prize, 2-40.

Parcells, C., Stommel, M. y Hubbard, R.P. (1999). Mismatch of classroom furniture and student body dimensions. Journal of Adolescent Health, 24(4), 265273, http://dx.doi.org/10.1016/S1054-139X(98)00113$\mathrm{X}$

Rea, M. S. (2000). The IESNA Lighting Handbook: Reference and Application. New York: The illuminating Engineering Society of North America.

Rittner, H. y Robbin, M. (2002). Color and light in learning. School Planning \& Management, 41(2), 5758.

Rivlin, L.G. y Weinstein, C.S. (1984). Educational issues, school setting, and environmental psychology. Journal of Environmental Psychology, 4(4), 347-364, http://dx.doi.org/10.1016/S0272-4944(84)80005-5

Scott-Webber, L., Strickland, A. y Kapitula, L.R. (2013). Built environments impact behaviours results of an active learning post-occupancy evaluation. Planning for higher education journal, 4(2), 1-12.

Spivack, J.A., Askay, A.D. y Rogelberg, G.S. (2009). Contemporary physical workspaces: A review of current research, trends, and implications for future environmental psychology inquiry. En J. Valentin, y L. Gamez (Eds.), Environmental psychology new developments. New York, Nova Science Publishers.

Taylor, A. (2009). Linking Architecture an Education: Sustainable design for learning environments. Alburquerque: University of New Mexico Press.

Torbert, J.B. (1987). A study of the physiological factors affecting the nature of the adult learner in the phoenix air National Guard. Nova University (ERIC Document Reproduction Service No. ED 284 044), http://eric.ed.gov/?id=ED284044.

Winterbottom, M. y Wilkins. A. (2009). Lighting and discomfort.Journal of Environmental Psychology, 29(1), 63-75,

http://dx.doi.org/10.1016/j.jenvp.2008.11.007 